\title{
The H.E.S.S. II GRB Program
}

\section{R.D. Parsons*}

Max-Planck-Institut für Kernphysik, Heidelberg, Germany

E-mail: daniel.parsons@mpi-hd.mpg.de

\section{A. Balzer}

GRAPPA, Anton Pannekoek Institute for Astronomy, University of Amsterdam, Amsterdam, The

Netherlands

E-mail: arnim.balzereuva.nle

\section{Füssling}

DESY, Zeuthen, Germany

E-mail: matthias.fuessling@desy.de

\section{Hoischen}

Institut für Physik und Astronomie, Universität Potsdam, Potsdam, Germany

E-mail: clemens.hoischen@desy.de

\section{Holler}

Laboratoire Leprince-Ringuet, Palaiseau, Ecole Polytechnique, CNRS/IN2P3, France

E-mail: hollerallr.in2p3.fr

\section{A.M.W. Mitchell}

Max-Planck-Institut für Kernphysik, Heidelberg, Germany

E-mail: alison.mitchellempi-hd.mpg.de

\section{G. Pühlhofer}

Institut für Astronomie und Astrophysik, Universität Tübingen, Tübingen, Germany

E-mail: Gerd.Puehlhoferdastro.uni-tuebingen.de

\section{G. Rowell}

School of Chemistry \& Physics, University of Adelaide, Adelaide, Australia

E-mail: gavin.rowelleadelaide.edu.au

\section{S. Wagner}

Landessternwarte, Universität Heidelberg, Königstuhl, Heidelberg, Germany

E-mail: swagneralsw.uni-heidelberg.de

\section{E. Bissaldi}

INFN - Sez. di Bari, Via E. Orabona 4, Bari, Italy

E-mail: Elisabetta.Bissaldi@uibk.ac.at

\section{P. O’Brien}

Department of Physics and Astronomy, University of Leicester, Leicester, United Kingdom E-mail: paul.obrienaleicester.ac.uk

\section{P.H.T. Tam}




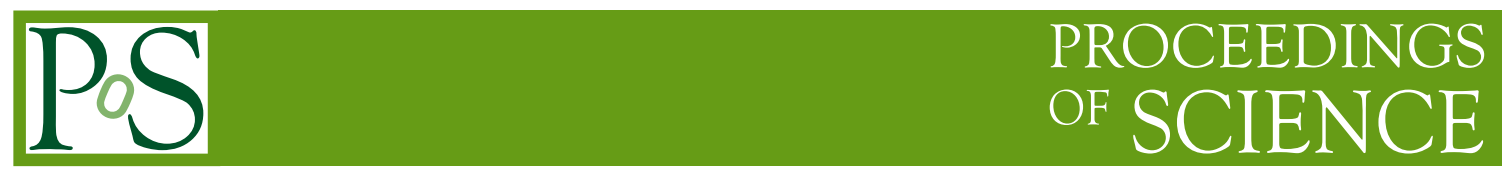

Institute of Astronomy and Space Science, Sun Yat-Sen University, Guangzhou, China E-mail: tanbxuandsysu.edu.cn

\section{on behalf of the H.E.S.S. Collaboration}

Gamma-ray bursts (GRBs) are some of the most energetic and exotic events in the Universe, however their behaviour at the highest energies $(>10 \mathrm{GeV})$ is largely unknown. Although the Fermi-LAT space telescope has detected several GRBs in this energy range, it is limited by the relatively small collection area of the instrument. The H.E.S.S. experiment has now entered its second phase by adding a fifth telescope of $600 \mathrm{~m}^{2}$ mirror area to the centre of the array. This new telescope increases the energy range of the array, allowing it to probe the sub-100 GeV range while maintaining the large collection area of ground based gamma-ray observatories, essential to probing short-term variability at these energies.

We will present a description of the GRB observation scheme used by the H.E.S.S. experiment, summarising the behaviour and performance of the rapid GRB repointing system, the conditions under which potential GRB repointings are made and the data analysis scheme used for these observations.

The 34th International Cosmic Ray Conference,

30 July- 6 August, 2015

The Hague, The Netherlands

${ }^{*}$ Speaker. 


\section{Introduction}

GRBs are the most luminous, highly-relativistic light sources known and may be generated during the collapse of a massive star (long GRBs) or via a merger event (short GRBs). They emit light across the electromagnetic spectrum, including at $\mathrm{GeV}$ energies (e.g. [1]) and hence provide key targets for very high energy (VHE) gamma-ray detectors, such as H.E.S.S. (e.g. [2, 3, 4, 5]). During the acceleration and emission period, GRBs may also be sources of ultra-high-energy cosmic rays (UHECRs; [6]), in addition to gravitational waves and neutrinos. Understanding the properties of GRBs therefore permit multiple science objectives to be addressed simultaneously. These include: (a) determine the physics of the jet including the outflow speed and emission site; (b) search for hadronic spectral signatures to explore jet composition and test the UHECR origin; (c) constrain the particle acceleration mechanism and radiation process(es); (d) measure the gammagamma attenuation due to the extra-galactic background light; and (e) test for Lorentz invariance violation (e.g. [7]). These objectives require high photon rates and time-resolved spectroscopy, preferably extending into the GeV-TeV band.

The H.E.S.S. gamma-ray telescope array has recently been upgraded by the addition of a fifth, $600 \mathrm{~m}^{2}$ telescope to the array centre (CT 5). This large telescope greatly reduces the energy threshold of the array. Together with the large effective collection area, the upgrade has greatly increased the chance for GRB detection. The synergy of H.E.S.S. phase II with other multi-messenger facilities would allow us to study GRBs in unparalleled ways.

\section{The H.E.S.S. II Rapid Repointing System}

As the gamma-ray luminosity of GRBs falls off very rapidly after burst time, in order to maximise the chances of a GRB detection at very high energies it is crucial to begin observations as soon as possible. In order to minimise this delay two major improvements have been made for the additional telescope (CT 5) over the original 4 telescopes. Firstly the telescope drive system of CT5 is significantly updated over that of the original H.E.S.S. system (see [8] for details), and is able to perform a full rotation of the telescope $\left(180^{\circ}\right.$ in azimuth) in $\sim 110$ seconds. Additionally CT5 is permitted to point in reverse-mode, allowing the telescope to slew through zenith, resulting in significantly faster repointing for some GRBs, where otherwise a large azimuthal slew would be required (see Figure 1).

In addition to this rapid slewing a fully automatic target of opportunity (ToO) observation system has been implemented within the H.E.S.S. array, in this case receiving triggers from the $\mathrm{GCN}$ system. The purpose of this system is to minimise the system repointing time by reducing the number of hardware and software transitions required to take place before observations can take place (see [9] for a detailed explanation).

The full ToO system is checked by the injection of fake GRB alerts at known source positions on a monthly basis, in order to ensure the repointing scheme is working reliably and the fastest possible repointing is achieved. Figure 2 shows a log of the performance of the ToO system on real and fake GRBs, demonstrating the fast repositioning time and continual improvements to the system. 


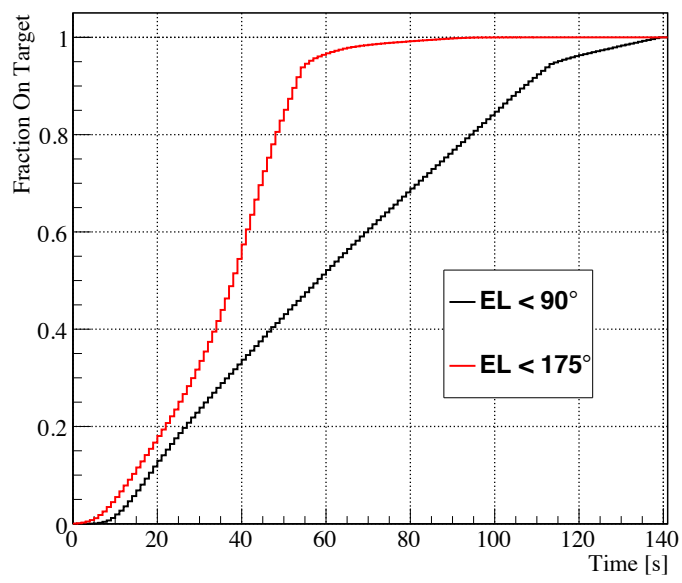

Figure 1: Fraction of times within which the CT5 telescope is able to arrive at a (random) target position on the sky versus the time after the issue of the repointing command. This fraction is shown for the systems with (elevation $<175^{\circ}$, red line) and without (elevation $<90^{\circ}$, black line) reverse-mode enabled. Figure reproduced from [8].

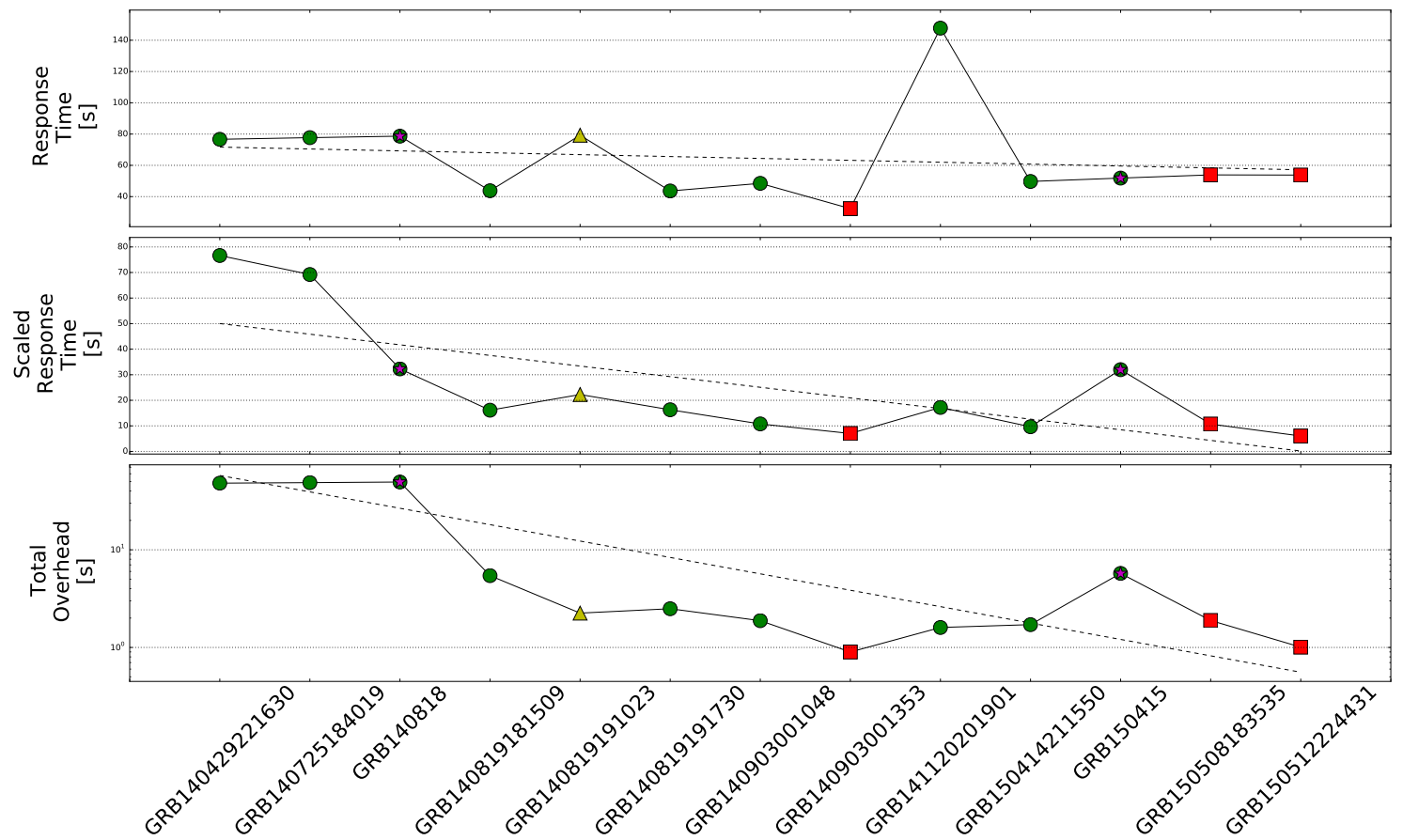

Figure 2: GRB observation total response time (top), response time scaled by the angular distance of the repointing (middle) and the data aquisition system time overhead (bottom) for a number of real and fake GRB observations. Figure adapted from [9]. 


\section{GRB Observations with H.E.S.S. II}

\subsection{The GCN System}

The H.E.S.S. array receives the notification of potential GRB repointings through the Gammaray Coordinates Network (GCN) [10]. The GCN system is designed to automatically distribute details of GRB detections and localisations from a number of instruments as rapidly as possible. Currently the H.E.S.S. array receives GRB localisations from 2 instruments, the Fermi-GBM and the Swift-BAT.

\subsubsection{Swift Telescope}

The Swift telescope is a multi-instrument telescope, launched in 2004, capable of rapid GRB triggers and follow-ups. The Burst Alert Telescope (BAT) [11] is a coded mask gamma-ray detector sensitive in the $15-150 \mathrm{keV}$ range. The BAT covers an angular range of almost 1.4 steradians, and is able to provide localisations with a positional accuracy at the arcminute level, with roughly 90 GRBs detected per year. Typically bursts are followed up by the other instruments on the Swift telescope; the UV optical telescope (UVOT) and the X-ray telescope (XRT), which provide spectra and light curves in these wavelength bands.

\subsubsection{Fermi Gamma-ray Space Telescope}

The Fermi Gamma-ray Burst Monitor (GBM) [12] consists of a series of scintillation detector placed around the Fermi gamma-ray space telescope providing almost all-sky coverage of gamma-ray transients in the $8 \mathrm{keV}$ to $40 \mathrm{MeV}$ range, reporting to the GCN system around 200 GRB localisations per year. Although this instrument has excellent sky coverage, unfortunately the accuracy of localisation is typically somewhat limited, measuring the position of the GRB to an accuracy of 2-5 degrees [13]. Such a large error box can be quite limiting when performing follow-up observations since it is is often of the order of the size of a typical IACT field of view.

\subsection{Selection Criteria}

Selection of GRB follow-ups with H.E.S.S. are made on a combination of observability based criteria and the likelihood of observable very high energy emission from the object. GRB follow-up observations are split into two categories, prompt and afterglow observations.

Prompt follow-ups are defined as those where the GRB occurs within H.E.S.S. darktime and is immediately observable. In this case the GRBs are subject only to observability criteria requiring that the GRB position is observable for a minimum of 30 minutes above a zenith angle of 60 degrees. Once an observation has begun, it will typically continue for 2 hours, or until the source drops below the 60 degree zenith threshold. If further, more accurate localisations are received within the observation time the observation position is updated automatically such that we are certain that the GRB is optimally placed within the field of view. The expected rate of prompt observation is around 4-6 per year of observations.

Conversely afterglow follow-ups are defined as GRB localisations observable from the H.E.S.S site, but not immediately at burst time. Afterglow observations are subject to more stringent observability cuts, requiring that the burst be observable above a zenith angle of 45 degrees for 30 
minutes or more. Additional cuts are placed on the delay between the burst time and the time at which the GRB becomes observable. In the most common case, where the redshift of the GRB is unknown a maximum of 4 hours delay is allowed, this allowed delay increases to 6 hours for sources with redshift below 1, 12 hours for redshifts below 0.3 , and 24 hours for redshifts below 0.1. In the case of afterglows a GRB expert is included in the decision making process, such that unusual or especially interesting bursts can still be chosen The expected rate of GRB follow-ups for these cuts is $8-12$ observations per year.

\section{Data Analysis Description}

\subsection{Data Blinding}

GRBs present an unusual observational situation for imaging atmospheric Cherenkov telescopes (IACTs) in that they are transient sources which in some cases may have a rather poor positional accuracy. When searching for these sources we must be careful not to introduce a large number of statistical trials while also being certain that any detections are not a false positive. Therefore, in order to ensure control of the trials factors the GRB observations are subjected to a blinding procedure. After data taking is complete no analysis of the data is made and the typical online data calibration and analysis is disabled, due to the large systematics present in the automated procedure. Instead the data is transferred to Europe blinded, where a Human in Loop data calibration can take place. However, efforts are ongoing to improve the low energy stability of the on-site analysis, which may allow an accurate automated data analysis in the future.

Once the data is calibrated each observation run is checked individually for known camera hardware and data calibration issues, to ensure that no spurious sources are generated in the final data analysis from these issues.

\subsection{Data Analysis}

Once the data has been fully calibrated and checked it can then be analysed using the H.E.S.S. II analysis pipeline. Currently events are split into to two classes within the H.E.S.S. II system, mono-events which are seen only by CT 5 and stereo events which are seen by at least 2 telescopes, these 2 classes can be analysed separately or joined into a single combined analysis (full analysis scheme described in [14]). As the emission spectrum of GRBs is expected to be quite steep, in this case the mono event analysis (which has the lowest energy threshold) is expected to be the most important. More details of the individual H.E.S.S. II analysis schemes can be found in $[15,16,17]$.

\section{Conclusions}

We have described the adaptations made to the H.E.S.S. II observation scheme to allow for the fastest possible follow-ups of GRB positions. Taking into account the delay of the GCN system, the hardware and software overheads, and the repointing time of the array we expect to arrive at the GRB target position within 2 minutes for the majority of possible observation positions. Analysis of simulated data shows that with the low energy optimised mono-event analysis we expect to be sensitive down to around $30 \mathrm{GeV}$, greatly overlapping with the observed range of energies already 
seen by Fermi-LAT [18], with a greatly increased effective area. This rapid response, in conjunction with good low energy performance should provide the H.E.S.S. array with a strong chance of a future GRB detection at very high energies!

Acknowledgment:The support of the Namibian authorities and of the University of Namibia in facilitating the construction and operation of H.E.S.S. is gratefully acknowledged, as is the support by the German Ministry for Education and Research (BMBF), the Max Planck Society, the German Research Foundation (DFG), the French Ministry for Research, the CNRS-IN2P3 and the Astroparticle Interdisciplinary Programme of the CNRS, the U.K. Science and Technology Facilities Council (STFC), the IPNP of the Charles University, the Czech Science Foundation, the Polish Ministry of Science and Higher Education, the South African Department of Science and Technology and National Research Foundation, and by the University of Namibia. We appreciate the excellent work of the technical support staff in Berlin, Durham, Hamburg, Heidelberg, Palaiseau, Paris, Saclay, and in Namibia in the construction and operation of the equipment.

\section{References}

[1] M. Ackermann, M. Ajello, K. Asano. et al The First Fermi-LAT Gamma-Ray Burst Catalog. The Astrophysical Journal Supplement, Volume 209, 1,90, 2013.

[2] F. Aharonian, A.G. Akhperjanian, U. Barres de Almeida, et al. HESS observations of $\gamma$-ray bursts in 2003-2007. Astronomy and Astrophysics, Volume 495, 2, 505, 2009.

[3] J. Albert, E. Aliu, H. Anderhub, et al. MAGIC upper limits on the very high energy emission from GRBs. The Astrophysical Journal, Volume 667, 1, 358, 2007.

[4] E. Aliu, T. Aune, A. Barnacka, et al. Constraints on Very High Energy Emission from GRB 130427A. The Astrophysical Journal Letters, Volume 795, 1, 2014.

[5] A. U. Abeysekara, R. Alfaro, C. Alvarez, et al. Search for gamma-rays from the unusually bright GRB 130427A with the HAWC Gamma-ray Observatory. The Astrophysical Journal, Volume 800, 2, 78, 6, 2015.

[6] F. Halzen. Pionic photons and neutrinos from cosmic ray accelerators. Astroparticle Physics, 43, 155, 2013.

[7] A.A. Abdo, M. Ackermann, M. Ajello, et al. A limit on the variation of the speed of light arising from quantum gravity effects. Nature, 462, 7271, 331

[8] P. Hofverberg, R. Kankanyan, M. Panter, et al. Commissioning and initial performance of the H.E.S.S. II drive system. Proceedings of the 33rd International Cosmic Ray Conference, 2013.

[9] A. Balzer, M. Füssling, P. Hofverberg et al. The Performance of the H.E.S.S. Target of Opportunity Alert System. Proceedings of CHEP 2015.

[10] The Gamma-ray Coordinates Network http://gcn.gscfc.nasa.gov

[11] S. D. Barthelmy, L. M. Barbier, J. R. Cummings et al. The Burst Alert Telescope (BAT) on the SWIFT Midex Mission. Space Science Reviews, 120, 143-164, 2005

[12] C. Meegan, G. Lichti, P. N. Bhat, et al. The Fermi Gamma-Ray Burst Monitor. The Astrophysical Journal, Volume 702, 1, 791-804, 2009

[13] V. Connaughton, M. S. Briggs, A. Goldstein, et al. Localization of Gamma-Ray Bursts using the Fermi Gamma-Ray Burst Monitor. The Astrophysical Journal Supplement, Volume 216, 2, 27, 2015. 
[14] M. Holler et al. Observations of the Crab Nebula with H.E.S.S. Phase 2. Proceedings of the 34th International Cosmic Ray Conference, 2015

[15] M. Holler \& M. de Naurois. Photon Reconstruction for H.E.S.S. using a Semi-Analytical Model. Proceedings of the 34th International Cosmic Ray Conference, 2015

[16] R.D. Parsons, M. Gajdus, and T. Murach. H.E.S.S. II Data analysis with ImPACT. Proceedings of the 34th International Cosmic Ray Conference, 2015

[17] T. Murach, M. Gajdus, and R.D. Parsons. A Neural Network-based Reconstruction Algorithm for monoscopically detected Air Showers observed with the H.E.S.S. Experiment. Proceedings of the 34th International Cosmic Ray Conference, 2015

[18] M. Ackermann, M. Ajello, K. Asano et al. The First Fermi-LAT Gamma-Ray Burst Catalog. The Astrophysical Journal Supplement, Volume 209, Issue 1, article id. 11, 90 pp. (2013). 